\title{
Empatica E3 - A wearable wireless multi-sensor device for real-time computerized biofeedback and data acquisition
}

\author{
Maurizio Garbarino*, Matteo Lai ${ }^{\dagger}$, Dan Bender ${ }^{\ddagger}$, Rosalind W. Picard ${ }^{\S}$, and Simone Tognetti ${ }^{\Uparrow}$ \\ $* \dagger \ddagger \S$ Empatica, Inc, Cambridge, MA, USA and Milan, Italy \\ $\S$ Massachusetts Institute of Technology, Cambridge, MA, USA

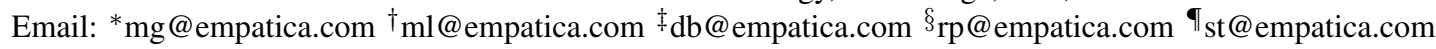

\begin{abstract}
The Empatica $\mathbf{E 3}$ is a wearable wireless multisensor device for real-time computerized biofeedback and data acquisition. The E3 has four embedded sensors: photoplethysmograph (PPG), electrodermal activity (EDA), 3-axis accelerometer, and temperature. It is small, light and comfortable and it is suitable for almost all real-life applications. The E3 operates both in streaming mode for real-time data processing using a Bluetooth low energy interface and in recording mode using its internal flash memory. With $\mathbf{E 3}$, it is possible to conduct research outside of the lab by acquiring continuous data for ambulatory situations in a comfortable and non-distracting way.
\end{abstract}

Keywords-wearable sensor, autonomic stress measurement, heart-rate, skin conductance, EDA, GSR

\section{INTRODUCTION}

The Empatica E3 (Figure 1) is a wearable wireless multisensor device for real-time computerised biofeedback and data acquisition. The E3 wristband has four embedded sensors: photoplethysmograph (PPG) (Figure 3), electrodermal activity (EDA) (Figure 2), 3-axis accelerometer and temperature sensor (Figure 5). The package is small $(4 \mathrm{~cm} \times 4 \mathrm{~cm})$, lightweight and comfortable and suitable for almost all real-life applications. (It is not yet suitable for submersion in water.) The E3 can operate in streaming mode for real-time data processing using a Bluetooth 4.0 (Bluetooth Low Energy - BLE) interface and in-memory recording mode using its internal flash memory. With the E3 wristband, it is possible to conduct health research outside of the lab by acquiring continuous data for ambulatory recording in a comfortable and compact wearable form. Though a number of wearable health devices exist in the market, the E3 with Empatica software and services offers the only device capable of providing quality continuous data representing the two main branches of the autonomic nervous system stress response.

\section{END USER AND RESEARCHER BENEFITS}

The end user is intended as the actual subject wearing and using the E3 wristband during the experiment. The main advantage for the end user is the E3 form factor, which efficiently combines 4 sensors into the wristband, replacing traditional multiple sources (e.g., heart rate chest strap, finger-placed EDA sensor, wrist worn accelerometers and temperature). Unlike traditional physiological acquisition devices, the E3 wristband can be worn during daily activities that include washing the hands; therefore, the wristband is less likely to interfere with

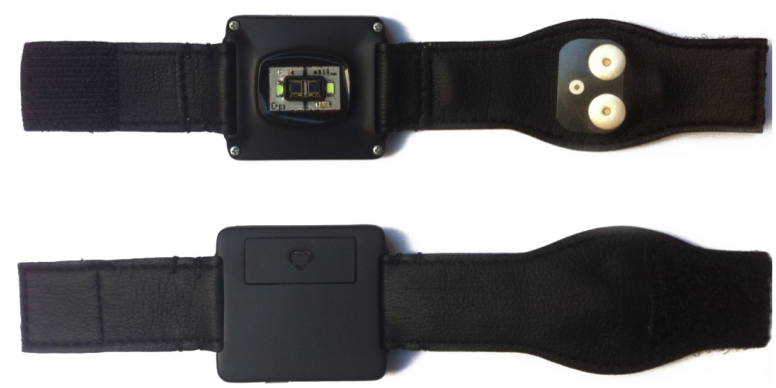

Fig. 1: Bottom and top view of E3 wristband

everyday activities. The E3's sensors are integrated into the band preventing the need for external leads. The rechargeable battery lasts up to 38 hours while it logs raw data from all four sensors in memory supporting more than a day and a half of continuous acquisition, or multiple shorter sessions.

The researcher benefits from the E3 wristband in many ways. The user experience is simplified making experimental setup easy. The wireless form factor supports validation of lab-based research in participants' natural environments.

\section{DATA QUALITY}

What distinguishes the E3 from other wearable devices is the full set and quality of data that it provides. The E3 has 4 integrated sensors: The PPG and EDA have a proprietary design while the 3 -axis accelerometer and the infrared thermopile are off-the-shelf components. While it is possible to find a number of other wearable devices that measure data related to health, notably the Biopac Nomadix, the Affectiva Q sensor, the Actigraph, the Mio Alpha, the Basis B1, CamNtech Actiheart and the Bodymedia LINK, they do not provide the same set of high-resolution quality autonomic sensors as the E3.

The E3 provides two significant advances in data quality. The first improvement relates to the PPG sensor, which uses a proprietary artifact removal technique based on a combination of multiple wavelengths. This technique is different from the common technique that exploits a single wavelength together with accelerometer information [1]. The second improvement relates to the EDA sensor and its dynamic range that allows 
an unusually high range of values $[0,100] \mathrm{uS}$ with a resolution of 900 picoSiemens. While EDA does not usually go above the bottom quartile of this range in lab studies, our team has observed the upper half of the range being reached in real-world medical events such as seizures. This range and resolution is more than satisfactory for recording the skin conductance level (SCL) and the skin conductance response (SCR) [2].

\section{A. Electrodermal Activity Sensor}

The E3 is equipped with an EDA sensor. EDA [3] is usually measured via the electrical conductance of the skin. Since sweat glands are controlled by the sympathetic nervous system, EDA can be used as an indication of psychological arousal.

EDA can sometimes vary significantly among different sites of measurement (e.g., fingers, palm, and wrist) [4], although in most cases the EDA measures made at the wrist are correlated with the traditional placements [5], [6]. However, in some cases, depending on which neurological system is activated in the brain, different values of EDA might be measured from left and right wrist [7].

In order to optimise both comfort and signal quality, the E3 is equipped with a peripheral board for EDA circuitry that, when worn, it is placed on the ventral area of the wrist. The terminal part of the EDA sensor is composed of two silver-coated (Ag) electrodes. A small alternating current is applied to the skin through the electrodes. The Ag electrodes are hypoallergenic and durable, and the E3 supports periodic electrode replacement and affords researchers the opportunity to use custom electrode materials or lead wire extensions to support traditional electrode placement, if desired.

Electrodermal activity is commonly measured in micro Siemens. In general, the higher the sweat gland activity, the higher the skin conductance value. The EDA is typically described as a combination of two components, the SCL and the SCR. The E3 has an innovative technology that accurately records SCL while maintaining sufficient sensitivity to distinguish the SCR under any condition. High resolution EDA (skin conductance) data can be used by researchers for measuring sympathetic activation / autonomic stress.

The E3 EDA sensor can measure conductance in the $[0.01,100] \mathrm{uS}$ range with a default sampling rate of $4 \mathrm{~Hz}$. The digital resolution is 1 digit per 900 picoSiemens. Such resolution is enough to sense EDA variations even in young children and elderly people, as long as electrodes are properly placed and the skin is reasonably hydrated and healthy. When using dry electrodes, we recommend that an active task be first given to the participant (such as going for a brisk walk) and sufficient time allowed to build up adequate moisture where the skin contacts the electrodes so that the electrodes record sensitive changes. The time needed can vary a lot, from seconds for a person with high hydration, to hours for a person with very dry skin in dry air or inactive conditions. Also, it may be the case that a person is highly responsive on one wrist, and not responsive on the other wrist [7]. In Figure 2 an example of a typical EDA signal is depicted. Three levels of zoom (5 hours, 30 minutes, 4 minutes) are reported to show the high dynamic range and sensitivity of the sensor.

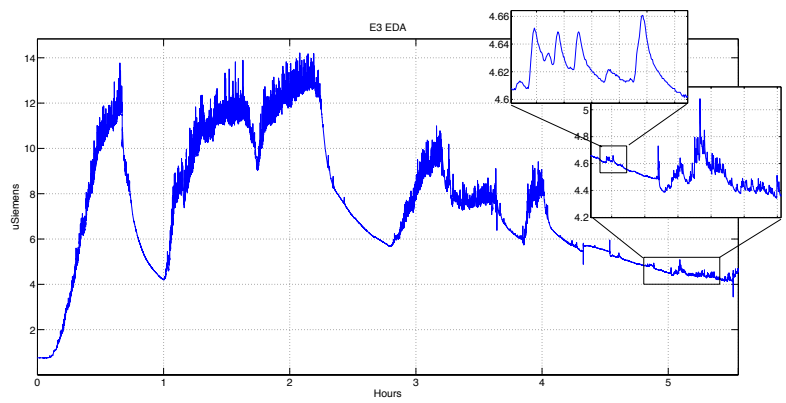

Fig. 2: Typical EDA signal. Three levels of zoom (5 hours, 30 minutes, 4 minutes) are reported to show the high dynamic range of the sensor.

\section{B. Photoplethysmography Sensor}

The E3 is equipped with a PPG sensor that illuminates the skin and measures the light reflected [8]. With each cardiac cycle, the heart pumps blood to the periphery. The change in volume caused by the pressure wave produced by the heart beat is correlated to a change in the concentration of oxyhemoglobin. Oxyhemoglobin absorbs light at certain wavelengths; therefore, the more oxyhemoglobin is present in the blood, the more light is absorbed. Each cardiac cycle appears as a peak of light absorption, as seen in Figure 3.

Standard techniques for artifact removal in wrist-worn PPG systems make use of accelerometers [1], [9]. Though, such methods give optimal performance when there is a repetitive movement that affects the sensor (e.g., walking, running), which can be modeled and compensated, they are not accurate when movement is sporadic and irregular. The PPG sensor of the E3 exploits a secondary source of light for tracking wrist movements in order to correct the artifacts obtained from the primary source of light. This technique also gives improved performance (over the traditional method) in situations where no significant wrist activity is observed from accelerometers (e.g., typing). Thus, the E3's custom PPG sensor can attenuate noise even when there are no repetitive movements to model.

The E3 photoplethysmography sensor samples at $64 \mathrm{~Hz}$. It is made of 4 light emitting diodes ( 2 green, 2 red), and 2 photodiodes (14 $\mathrm{mm}^{2}$ of sensitive area). The digital output of the sensor is the variation of light intensity with $0.9 \mathrm{nW} /$ Digit resolution. Differences in skin color and external light intensity are dynamically compensated by firmware.

Examples of the E3 PPG data are in Figure 3 (a) static and (b) with motion. The signal plotted is a zero mean signal with waves that indicate a variation of light due to the cardiac cycle. The resolution of this signal is $0.9 \mathrm{nW}$. Figure 3 highlights also the output of the beat detection algorithm provided by the E3. The inter-beat interval output allows the researcher to conduct heart rate variability analysis [10], which is important in estimating vagal tone and parasympathetic nervous system activity.

Figure 4 depicts a 38 hour continuous recording of heart rate provide by the $\mathrm{E} 3$. 


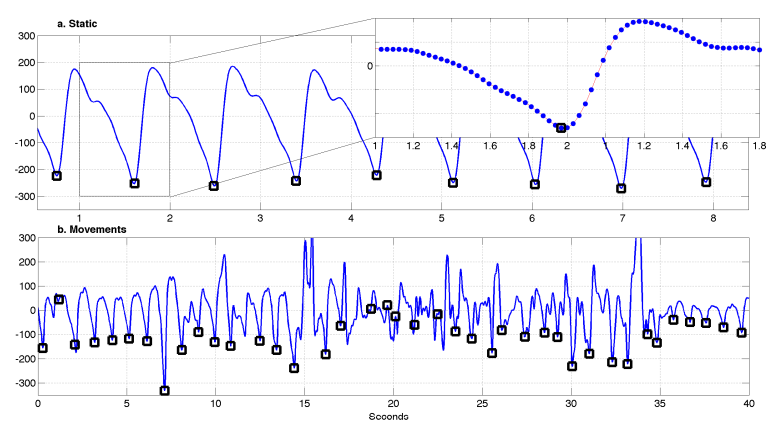

Fig. 3: Examples of the PPG data (a) static and (b) during arm movement. The signal plotted is a zero mean signal with waves that indicate a variation of light due to the cardiac cycle.

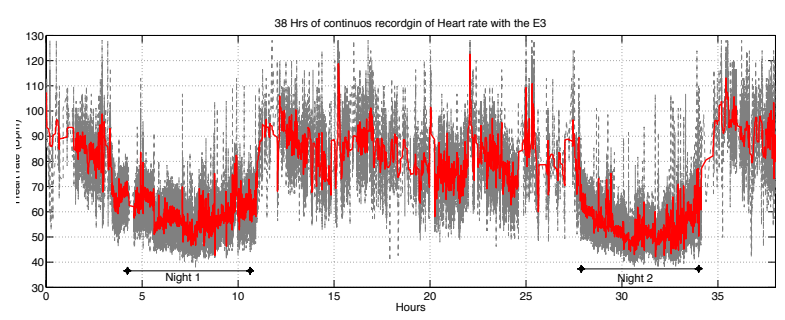

Fig. 4: Thirty eight hours of continuos heart rate monitoring. Note the lower heart rate during the two nights.

\section{Temperature Sensor}

Skin temperature is recorded with an optical infrared thermometer located between the EDA electrodes. Advantages of such a technology are the possibility to perform non-contact measurements, a fast response compared to traditional contact sensors, and a stable calibration over time. To avoid biases in the measurement, the sensor is placed in contact with the skin and is surrounded by dark material to reduce external disturbances. With regard to emissivity, the skin ranges around 0.97 for infrared radiation ( 1 is maximum); therefore, it is correctly measured by an optical sensor. Whereas the provided resolution for the sensor is $0.02{ }^{\circ} \mathrm{C}$, the accuracy over the range of human skin temperature is $\pm 0.2{ }^{\circ} \mathrm{C}$. Calibration is valid in the range $[-40,115]^{\circ} \mathrm{C}$.

The temperature sensor of the E3 also allows measurement of the ambient temperature (Figure 5) from the sensor case. This is essentially a low pass version of the body temperature, although the difference between the two temperatures is not always constant. Situations that cause vasoconstriction might cause the skin temperature to drop more rapidly than the ambient or case temperature. Thus, measuring both temperatures can give valuable information.

\section{Three-Axis Acceleration Sensor}

The accelerometer is configured with a sample frequency of $32 \mathrm{~Hz}$ and measures 3 axes, $\mathrm{X}, \mathrm{Y}$ and $\mathrm{Z}$. Its default range is $\pm 2 \mathrm{~g}$ although ranges of $\pm 4 \mathrm{~g}$ or $\pm 8 \mathrm{~g}$ are selectable with custom firmware. The signal resolution is 8 bits of the selected range $[-127,128]$. Over the default range of $\pm 2 \mathrm{~g}$, this translates to

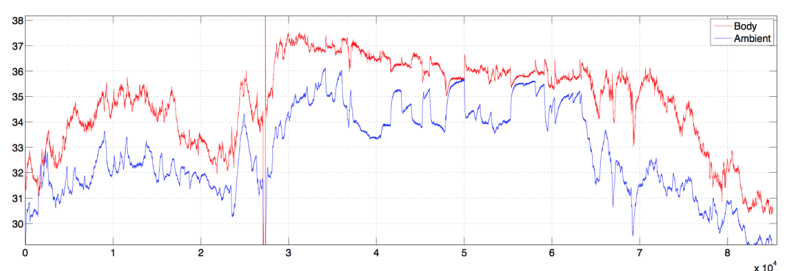

Fig. 5: Example of output generated by the E3 skin temperature (red), and ambient temperature (blue).

$0.016 \mathrm{~g}$ per count. Other selectable configurations are $8 \mathrm{bit} / \pm 4 \mathrm{~g}$, $8 \mathrm{bit} / \pm 8 \mathrm{~g}, \mathrm{~s} 12 \mathrm{bit} / \pm 2 \mathrm{~g}, 12 \mathrm{bit} / \pm 4 \mathrm{~g}, 12 \mathrm{bit} / \pm 8 \mathrm{~g}$.

\section{SOFtware ARchitecture}

In this section, an overview of the Empatica E3 software architecture (Figure 6) is described. The E3 wristband has 2 main working modalities: Realtime Streaming and In-memory Recording. Software is available for both desktop and mobile platforms, as well as for customising new mobile applications with multiple sensors.

In realtime streaming, the E3 wristband can connect via Empatica API to a mobile terminal (iOS, or Android platform) or to a computer via Bluetooth 4.0 (Bluetooth Low Energy BLE). An application for data acquisition is available from the mobile online stores to collect data in real time. At the end of the acquisition, sessions are uploaded to the Empatica server. With in-memory recording mode, data are stored into the internal flash memory and, at the end of the acquisition session, the wristband needs to be connected to a computer (PC or mac) via USB in order to upload data to the Empatica cloud server. After signal data acquired from the E3 sensors are uploaded to the Empatica cloud platform, signal processing software applies filters and extracts features from the raw signals (e.g., heart rate is extracted from the BVP signal). The raw data is also available for download as CSV files.

[width=260px]img/EmpaticaCloud.pdf

\section{A. Mobile - Empatica API}

API's are available for both iOS and Android platforms. The E3 Mobile API allows researchers to develop a custom mobile application specifically for their research. The API functions let the researcher handle simultaneous connection to multiple E3 devices. The application can subscribe to one or more of these signal channels: BVP, IBI, EDA, accelerometer, skin temperature. The application periodically receives realtime data packets containing information about timestamp and sample value. The E3 transmits data via BLE. The E3 mobile API currently supports the latest iOS and Android devices having BLE connection capabilities.

\section{B. Mobile - Realtime Streaming App}

The E3 realtime streaming app allows the user to visualise health and activity data from the wristband in realtime on mobile devices. A zoomable and pannable realtime plot for BVP and EDA signals is provided. Heart rate and skin temperature are displayed in numeric format. Raw data from the wristband 


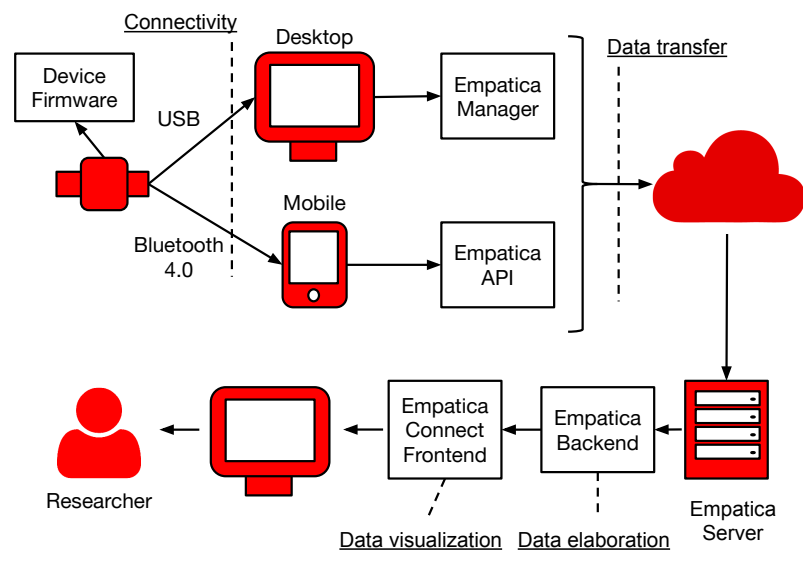

Fig. 6: Overview of Empatica E3 software architecture. Data automatically acquired from the device can be accessed by the researcher via the online Empatica website.

are collected and saved locally. At the end of a session, a raw data file is uploaded to the Empatica cloud server. The E3 realtime streaming app is available in the app store and currently supports the latest iOS and Android devices having BLE connection capabilities.

\section{Desktop - TCP Server}

The E3 TCP Server is an application that allows the development of custom software solutions that receive realtime data from multiple E3 devices. The server acts as a proxy between E3 devices and as researcher's custom applications (clients). The server accepts TCP socket connections. Clients are allowed to run discovery for new E3 devices and to connect to discovered E3 devices. After a successful connection, clients can send messages to the server to subscribe to one or more signal channels. The protocol is text based; data packets contain timestamp, sample type, and sample value. The current version of the TCP server only supports OSX but clients from any platform (e.g., windows, Android, iOS) can connect.

\section{Desktop - Empatica Manager}

The in-memory recording continuously records raw signals in the onboard E3 memory. After recording, the data are uploaded to the Empatica cloud by first plugging them into a Mac or PC via USB. The Empatica Manager is a desktop memory sync program that performs this operation automatically as soon as the user has logged in and connected a sensor. Data upload is secure and does not include personally identifying information, allowing the system to satisfy both USA and EU HIPPA requirements.

\section{E. Empatica Connect}

The Empatica Connect web platform is a place where the researcher can review and access all the sessions recorded with the E3 wristbands associated to her account. Data from each session can be visualised or downloaded in timestamped CSV format, making it easy to import into any data analysis tools.

\section{SUMMARY: IMPROVING DATA COLLECTION}

The E3 provides a new wrist-wearable sensor that compactly measures data characterising healthy autonomic nervous system function - EDA for sympathetic activation and PPG for deriving heart-rate variability for parasympathetic activation - together with temperature and activity. We have described performance features and demonstrated high data resolution and range, better than other wearables available.

The infrastructure described above has made adopting the E3 feasible for health researchers around the globe. The device has been tested and passed international safety and emissions standards, and has been approved by institutional review boards in top hospitals in the EU and USA. The E3 is well suited for integration into existing clinical trials. Derivative data, including changes in autonomic responsivity, sleep duration, temperature, and activity level are used for experimental outcome measures to inform therapy developers about objective impacts on patients. Clinical researchers today use Empatica Manager to sync data nightly and to verify protocol compliance while keeping subjects blinded to treatment condition.

The combined Empatica sensor and software utilities allow many new opportunities for researchers to build custom applications for mobile health, as well as to collect important health data in daily life from patients.

\section{REFERENCES}

[1] P. Renevey, R. Vetter, J. Krauss, P. Celka, and Y. Depeursinge, "Wristlocated pulse detection using ir signals, activity and nonlinear artifact cancellation," in Engineering in Medicine and Biology Society, 2001. Proceedings of the 23rd Annual International Conference of the IEEE, vol. 3. IEEE, 2001, pp. 3030-3033.

[2] W. T. Roth, M. E. Dawson, and D. L. Filion, "Publication recommendations for electrodermal measurements," Psychophysiology, vol. 49, pp. 1017-1034, 2012

[3] W. Boucsein, Electrodermal activity. Springer, 2012.

[4] A. S. Scerbo, L. W. Freedman, A. Raine, M. E. Dawson, and P. H. Venables, "A major effect of recording site on measurement of electrodermal activity,” Psychophysiology, vol. 29, no. 2, pp. 241-246, 1992.

[5] M.Z. Poh, N. C. Swenson, and R. W. Picard "A wearable sensor for unobtrusive, long-term assessment of electrodermal activity," Biomedical Engineering, IEEE Transactions on, vol. 57, no. 5, pp. 1243-1252, 2010.

[6] M. van Dooren, J. De Vries, and J. H. Janssen, "Emotional sweating across the body: Comparing 16 different skin conductance measurement locations," Physiology \& behavior, vol. 106, no. 2, pp. 298-304, 2012.

[7] R. W. Picard, S. Fedor, and Y. Ayzenberg, "Multiple arousal theory and daily-life electrodermal activity asymmetry," Emotion Review, 2014, to appear.

[8] J. Allen, "Photoplethysmography and its application in clinical physiological measurement," Physiological measurement, vol. 28, no. 3, p. R1, 2007.

[9] B. Lee, J. Han, H. J. Baek, J. H. Shin, K. S. Park, and W. J. Yi, "Improved elimination of motion artifacts from a photoplethysmographic signal using a kalman smoother with simultaneous accelerometry," Physiological measurement, vol. 31, no. 12, p. 1585, 2010.

[10] M. Malik, J. T. Bigger, A. J. Camm, R. E. Kleiger, A. Malliani, A. J. Moss, and P. J. Schwartz, "Heart rate variability standards of measurement, physiological interpretation, and clinical use," European heart journal, vol. 17, no. 3, pp. 354-381, 1996. 